\title{
Lessons Learned from 27 Years Experience and Focus Operating on Symptomatic Conditions of the Spine under Local Anesthesia: The Role and Future of Endoscopic Spine Surgery as a "Disruptive Technique" for Evidenced Based Medicine
}

Anthony T. Yeung*

Desert Institute for Spine Care, Phoenix, Arizona, USA

\begin{abstract}
The Practice of Medicine will always be an art based on science. The human body is more complex and not like a mathematical formula. History has shown that concepts in medicine, judged by "key opinion" leaders, currently depend on scientific publications that affect medical treatment through the medical literature. Strict adherence to Cochrane criteria and old "evidence based" concepts are used for scientific dissemination and publication, but also used for insurance reimbursement in the United States. Health care is becoming more and more supported by government subsidy and payment is dependent on guidelines established by each payer. The ability of innovative and valuable level five expert EBM opinions to get published for dissemination to the scientific community can be difficult for researchers because institutional support or NIH funding is the usual pathway. If there is no institutional support, researchers must pay fees to get their work published in open access Journals. It may be the time to consider "innovative disruption" as a form of evidence based medicine to mitigate the unsustainable increasing cost of health care for spine treatment. Endoscopic spine surgery fits consideration as an innovative disruptive procedure. Patients seeking advice from their chosen physician or health care provider for their physical complaints are for conditions that are based on the Physiology and Patho-anatomy causing their symptoms. In spinal conditions, while most patients are not taken seriously until they complain of "debilitating" pain, symptomatic conditions that can be resolved with tincture of time and/or supportive modalities are supported by allopathic as well as homeopathic and naturopathic physicians. Surgical or invasive procedures are usually reserved for more specific allopathic conditions. These conditions may cause not just pain, but numbness, a change in sensation, weakness, or only intermittent debilitation. The symptoms may also reflect separate and concomitant symptoms that can be confusing when the physician focuses on a single source of the symptom. Extensive experience and perseverance with techniques that work for the clinician is valuable when there is a database large enough to be studied and mined, to demonstrate statistical significance. Such is the case with procedures that can be validated by endoscopic imaging to evaluate, and validate the complaint by the ultimate result: symptom resolution. Traditional radiologic Imaging by itself, however, is inadequate to explain complaints of symptoms that may or may not be debilitating in the physician's judgment, and the patient may be simply dismissed or prescribed a drug to mitigate the complaint. This has, in many ways, contributed to the myriad of pharma solutions to every symptom complaint in allopathic medicine. A myriad of Naturo-pathic remedies are also marketed and sold over the counter, supported by millions of symptom sufferers who do not expect insurance reimbursement. There are also allopathic means to diagnose and treat symptomatic conditions in the spine, especially in the lumbar spine that currently garners third party reimbursement. The role and future of endoscopic spine surgery is supported here as an innovative disruption to our current means for scientific validation. Performed expertly and properly, a "warrantee" can be provided.
\end{abstract}

Keywords: Evidence based medicine; Endoscopic spine surgery; Local anesthesia, Pathophysiology and patho-anatomy of pain; Diagnostic and therapeutic injections; Evocative pre-operative discography; Intra-operative chromo-discography

\section{Introduction}

The Practice of Medicine will always be an Art, based on science, but rigid concepts in medicine, judged by key opinion leaders with emphasis on scientific publications, are affecting the medical literature through strict adherence to Cochrane criteria and "evidence based" concepts in order to be accepted for publication and reimbursement. Payers of Insurance re-imbursement does not recognize expert opinion but requires methods proven with level I or 2 evidence based studies [1]. Even if the result is warranted, insurance reimbursement to honor contracts that does not meet the arbitrary criteria of the insurance carrier needs reform. The ability of level five expert EBM opinions to get information to the scientific audience can also be difficult for most researchers because they either need institutional or NIH funding, or must pay publishing fees to get their work published by open access Journals. The best level I studies are sometimes ignored when the business of spine adversely determines the procedure's ultimate fate. Chymopapain is one such example [2].

\section{Literature Review}

Symptoms of patients in medicine on conditions based on the Physiology and Patho-anatomy causing symptoms, can be endoscopically validated. While most patients are sometimes only taken seriously only when they complain of debilitating pain, symptomatic conditions that can be resolved surgically may only cause numbness, a change in sensation, weakness, or intermittent debilitation [3]. The symptoms may also reflect separate and concomitant symptoms that can be confusing when allopathic physicians only focus on a single

*Corresponding author: Anthony T. Yeung, Desert Institute for Spine Care Phoenix, Arizona, USA, Tel: +1 602-944-2900; E-mail: ayeung@sciatica.com

Received April 16, 2017; Accepted April 23, 2017; Published April 27, 2017

Citation: Yeung AT (2018) Lessons Learned from 27 Years' Experience and Focus Operating on Symptomatic Conditions of the Spine under Local Anesthesia: The Role and Future of Endoscopic Spine Surgery as a "Disruptive Technique" for Evidenced Based Medicine. J Spine 7: 413. doi: 10.4172/2165-7939.1000413

Copyright: (C) 2017 Yeung AT. This is an open-access article distributed under the terms of the Creative Commons Attribution License, which permits unrestricted use, distribution, and reproduction in any medium, provided the original author and source are credited. 
Citation: Yeung AT (2018) Lessons Learned from 27 Years' Experience and Focus Operating on Symptomatic Conditions of the Spine under Local Anesthesia: The Role and Future of Endoscopic Spine Surgery as a "Disruptive Technique" for Evidenced Based Medicine. J Spine 7: 413. doi: 10.4172/2165-7939.1000413

Page 2 of 5

source of the symptoms. Extensive experience and perseverance with techniques that work for the clinician are required to establish a database large enough to be mined and to prove statistical significance. Such is the case with endoscopic imaging and the author's focus for the past 27 years.

The basics of trauma, tumor, and neuropathy are often present at the same time, and worsen with aging to the extent that it occurs in everyone, and physical immorality is still obviously not attainable [4]. The medical literature also reflects this basic "fact" as truth. Spinal conditions are an example where diagnostic and therapeutic injections can identify symptomatic conditions early, cost effectively, and safely. A few examples of lessons learned with endoscopic identification and treatment of these symptomatic conditions are illustrated with diagnostic and therapeutic injections, correlated with endoscopic imaging of the patho-physiology and path-anatomy of the symptom generator [5]. Some valuable diagnostic procedures are considered "controversial" because even evidence based publications have overt bias by key opinion leaders with different conclusions from published studies. This creates the "controversy" in the literature.

With over 27 years of experience in endoscopic spine surgery endoscopic spine surgeon Anthony Yeung presents his level 5 opinions gleaned from a review of greater than 10,000 of his cases since 1991, documented by his database of results backed by endoscopic images of patho-anatomy. This type of level $\mathrm{V}$ validation, together with surgery under local anesthesia allows the patient to provide real time feedback during and after surgery. This can be considered an "innovative disruption" to the usual evidenced based criteria used by payers for insurance reimbursement, but are increasingly accepted by cash patients $[6,7]$.

Endoscopic Imaging is relied on to evaluate and validate the patient's complaint. Imaging by itself, however, is inadequate to explain complaints of symptoms that may or may not be debilitating in the physician's judgment, and the patient may be simply dismissed or prescribed a drug to mitigate the complaint [8]. This has, in many ways, contributed to the myriad of pharma solutions to every symptom complaint, causing an opiod crisis when the focus is on pain. A myriad of Naturo-pathic remedies are marketed and not reimbursed by insurance that are sold over the counter. There are allopathic means to diagnose and treat symptomatic conditions in the spine, especially in the lumbar spine where endoscopic documentation is possible.

Symptoms are affected by conditions that may elude diagnosis and treatment because they are often combined conditions, or as a result of aging, that can exist together. This can be confusing to the physician clinician. In medical care, recent lobbying by pain physicians using pain as a $5^{\text {th }}$ vital sign has resulted in an opiod crisis when it is easiest to use drugs to mitigate pain. Such is the case when the investigation is for pain, weakness, and nerve mediated symptoms [9].

Symptoms of pain brings most patients to their physicians for treatment, but pain is a combination of inflammation and a condition causing inflammation that brings about this unpleasant sensation. Weakness can be the result of compression of peripheral nerves, the spinal cord, or arise in the central nervous system, the brain. All areas can be also affected by neoplasm from the complexities of cellular biology [10]. A healthy individual is constantly fighting cancerous cells constantly replicating in the natural process of healing and regeneration, but cancerous cells are usually destroyed early in the disease process by the auto-immune system in a healthy person. Ultimately, the immune system can be overwhelmed and fail, and auto immune conditions or neoplasms arise.
Peripheral neuropathy, a complex condition, will be affected by attempts at treating the nerve condition with surgical decompression, but the result may be affected by other non-surgical conditions. A condition known as sympathetic dystrophy, recognized to be a very difficult painful condition to treat, and can be the result of hyperactivity of peripheral nerve from the dorsal and ventral ramus [11]. Symptoms in individuals can result from multiple causes. Therefore, diagnostic and therapeutic injections often represent the first step in diagnosis and treatment.

Spinal conditions are an example where diagnostic and therapeutic injections, augmented by discography and foraminal epiduroscopy, can identify symptomatic conditions early, cost effectively, and safely. A few examples of lessons learned with endoscopic identification and treatment of these symptomatic conditions are illustrated, correlated with endoscopic imaging of not just the patho-physiology and pathanatomy of the symptom generator, but findings of anomalous anatomy are also discovered [12]. Over 27 years of experience in endoscopic spine surgery is presented. Anthony Yeung's procedure, philosophy and technique is presented here, reinforced by endoscopic treatment of a complex degenerative condition in the author's own spine culminating in the need for endoscopic and dynamic stabilization of his spine for degenerative scoliosis, spondylolisthesis, multi-modal disc herniations, lumbar spondylosis, and facet arthrosis [13]. The endoscopic procedures are shown on you tube videos of his surgical procedure performed by his son, Chris Yeung, a traditonally fellow shipped trained spine surgeon who is also endoscopically trained and using his father's Yeung philosophy and endoscopic technique at www. sciatica.com under the icon "watch real patient procedure videos."

\section{Discussion}

Anthony Yeung's surgery under local anesthesia without sedation

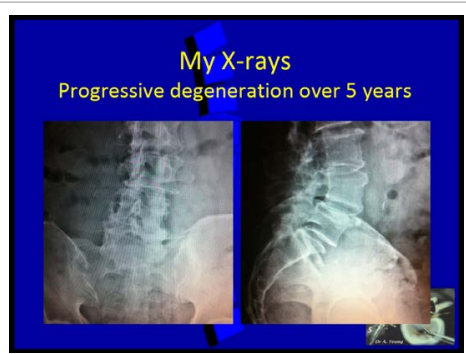

Figure 1: Prodromal symptoms progressing slowly over 2 years consisting of back ache with intermittent sciatica in the $L 5$ and $S 1$ distribution $R>L$ relieved by rest and Ibuprofen. In retrospect, treatment could and should have been considered earlier.

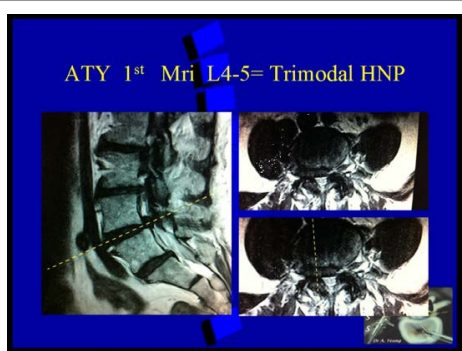

Figure 2: Yeung Experienced persistent sciatica 3 months before surgical intervention. 30 days before endoscopic decompression surgery, $3 / 5$ weakness with and EHL foot dorsiflexion, weak hip abduction, and inability to bear weight developed, and rapidly progressed. The MRI revealed a broad based Trimodal HNP L4-5 prompting the immediate decision for Endoscopic transforaminal decompression due to the neurological deficit. 
Citation: Yeung AT (2018) Lessons Learned from 27 Years' Experience and Focus Operating on Symptomatic Conditions of the Spine under Local Anesthesia: The Role and Future of Endoscopic Spine Surgery as a "Disruptive Technique" for Evidenced Based Medicine. J Spine 7: 413. doi: 10.4172/2165-7939.1000413

Page 3 of 5

is exhibited in a 3 part staged procedure described in this article. This personal experience with endoscopic surgery consolidated the elder Yeung's philosophy and technique through the successful treatment of his own condition, targeting the pain generator in a staged manner as an alternative to fusion (Figures 1-16).

These 2 examples represent the extremes of clinical conditions that can be treated by endoscopic decompression under local anesthesia. The database contains numerous examples of symptomatic conditions

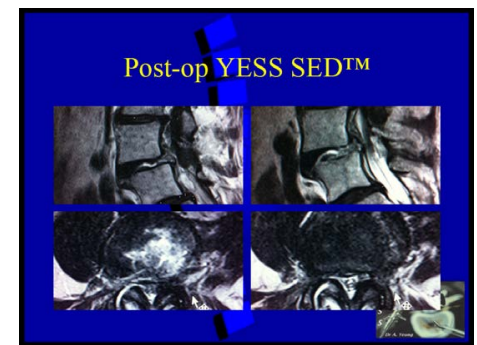

Note: Surgery was successful in relieving weakness and numbness, but a sharp pain persisted with spinal rotation. Instability and foraminal stenosis was apparent, so the decision to be made was fusion versus dynamic stabilization.

Figure 3: Post-op selective endoscopic discectomy ${ }^{\mathrm{TM}}$ lateral and axial view.

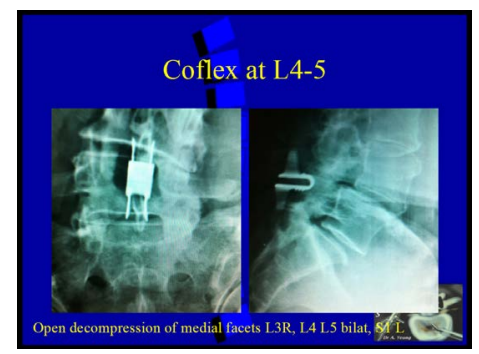

Note: Following his own surgical experience, Yeung systematically reviewed his philosophy and Technique from his $<10,000$ procedures that had endoscopic images saved since 1991 when he adopted endoscopic surgery. The following observations forming this opinion article are presented. Discography, epidurography, and therapeutic injections correlated well in predicting endoscopic surgical results focusing on intradiscal therapy through intradisca discectomy, irrigation, thermal ablation, and foraminal decompression.

Figure 4: Anthony Yeung chose additional translaminar decompression and Coflex dynamic stabilization over fusion and additional open decompression, recognizing that a fusion, if needed, can be staged. Yeung is now 4 years post op having successfully avoiding fusion.

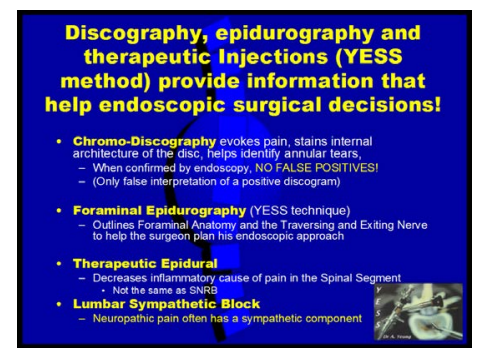

Note: Discography was especially valuable pre-operatively or intraoperatively when a chromophore was added at surgery to vitally stain degenerative nucleus for as a guide to the amount of disc to remove intradiscally under endoscopic visualization. There were virtually no false positives or negatives, only "false interpretation" in the early stages of endoscopic surgery. Different intradiscal pathologies were identified and treated.

Figure 5: This PPT slide summarizes the value of Intra-operative Chromodiscography, pre-operative foraminal epiduroscopy, therapeutic transforaminal epidural blocks and Lumbar sympathetic blocks that help predict and treat results in endoscopic spine surgery.

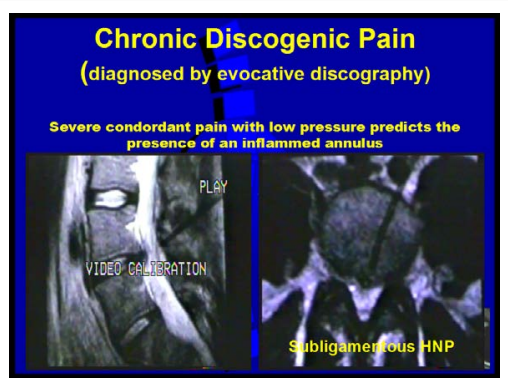

Figure 6: The value of pre-operative evocative discography in identifying the etiology of a painful disc.

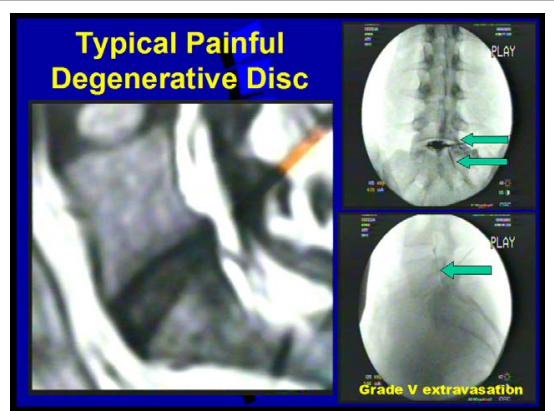

Note: When pain was out of proportion to the MRI image, grade IV and V annular tears were identified to leak to the dorsal root ganglion of the exiting nerve. Intradiscal thermal modulation of the tear after removal of interpositional nucleus material brought symptom relief. The length of pain relief was partly dependent on the extent of the tear, the thickness of the remaining annular layers, and the ability of the annulus to heal.

Figure 7: The value of discography in identifying a painful disc causing discogenic pain out of proportion to the image is demonstrated to be caused by irritation of the dorsal root ganglion responsible for pain out of proportion to the MRI image.

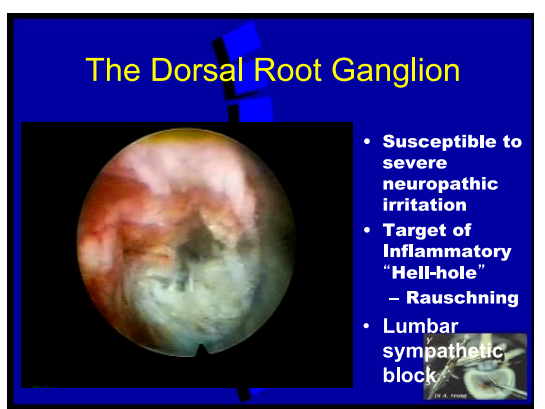

Figure 8: Inflammation around the dorsal root ganglion. The addition of an epidural gram as part of the pre-operative work-up also helped identify the patho-anatomy of HNP, central and lateral stenosis.

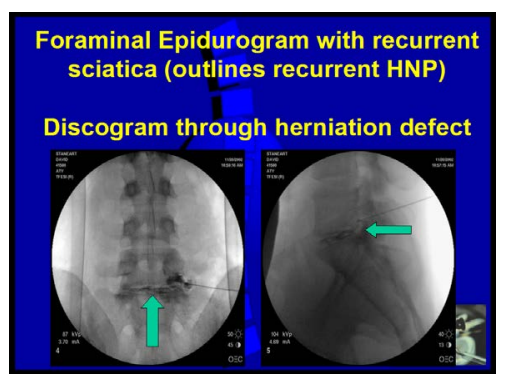

Figure 9: Incidental discogram identifying communication with the disc with an extruded HNP. Note the needle is cephalad to the disc space. 
Citation: Yeung AT (2018) Lessons Learned from 27 Years' Experience and Focus Operating on Symptomatic Conditions of the Spine under Local Anesthesia: The Role and Future of Endoscopic Spine Surgery as a "Disruptive Technique" for Evidenced Based Medicine. J Spine 7: 413. doi: 10.4172/2165-7939.1000413

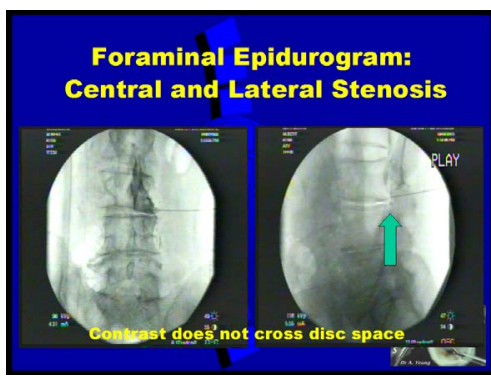

Note: Other symptomatic conditions were identified and correlated well to ultimately determine the results of foraminal decompression and intradiscal therapy. Examples ranging from painful annular tears to severe spinal and foraminal stenosis are illustrated by these illustrations of symptomatic conditions responding to endoscopic decompression from elderly patients with severe comorbitities creating a situation of risk vs. benefits.

Figure 10: An epiduralgram can also identify central plus lateral stenosis.

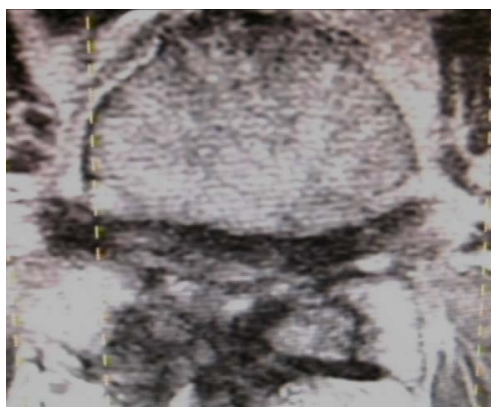

Figure 11: Clinical example \#1 Severe central stenosis, and intermitten claudication in an elderly man with high risk surgical morbidities who opted to try transforaminal decompression under local anesthesia.

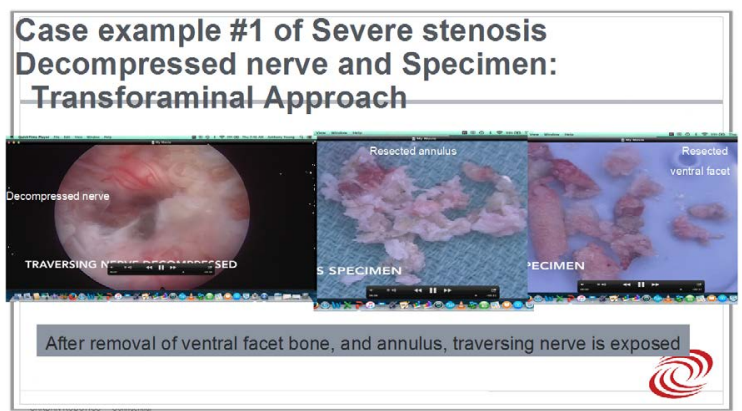

Figure 12: Case \#1 Intra-operative visualization of the decompressed traversing nerve. Surgical specimen of the resected dorsal annulus and the ventral facet after transforaminal removal.

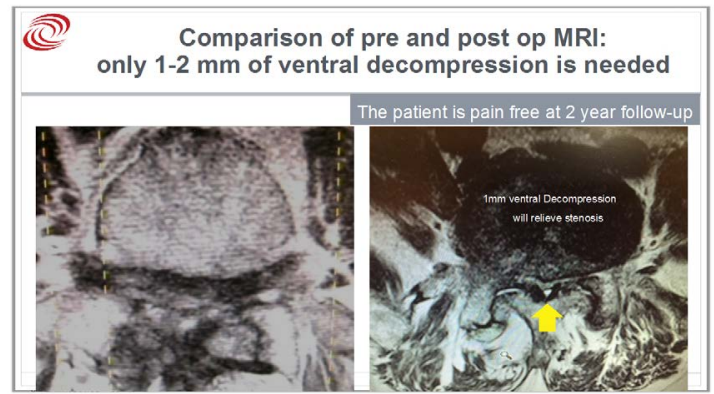

Figure 13: Case \#1 Only $1 \mathrm{~mm}$ of decompression is needed with ventral decompression under local anesthesia.

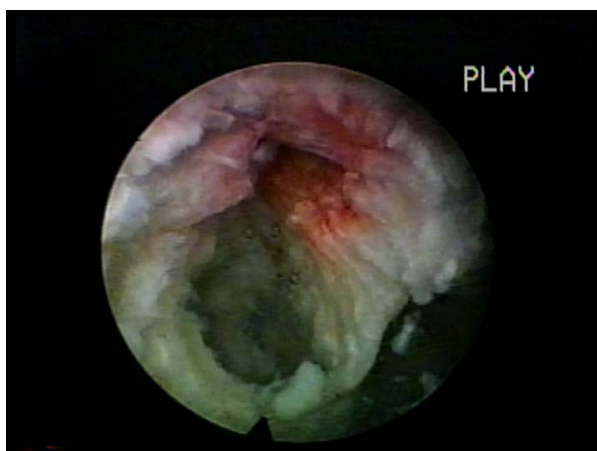

Figure 14: Clinical Example \#2 Severe discogenic pain from a toxic annular tear in the outer annulus at L5-S1.

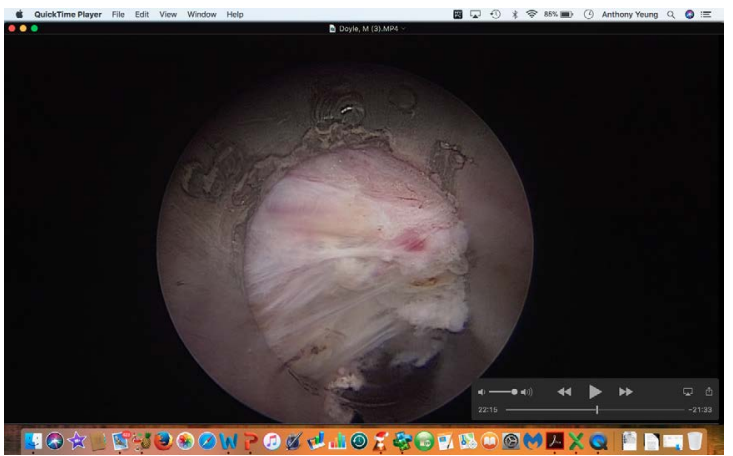

Figure 15: An inflammatory membrane containing tiny nerves can be seen in the foramen and epidural space. Occasionally small unmyelinated "sinuvertrebral" nerves are visualized. Ablation of these nerves may cause post-op dysesthesia that, if severe, will respond to transforaminal epidural and sympathetic blocks. If no pain is elicited, it can be part of the foraminal ligament or a branches from the dorsal ramus, a sensory nerve.

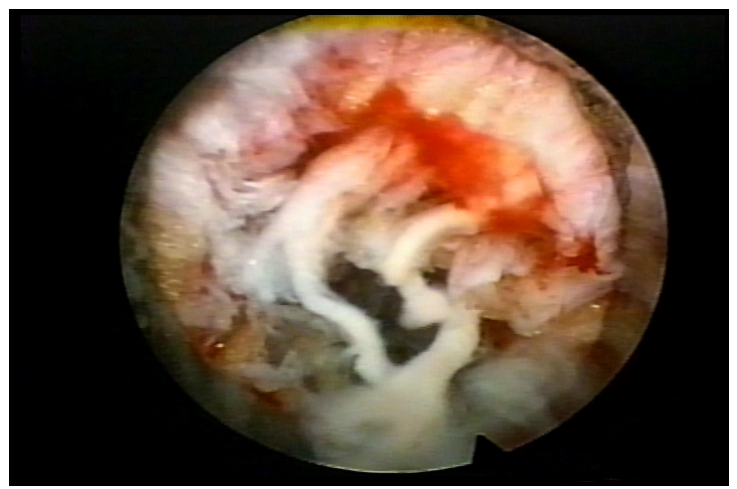

Figure 16: Sensory nerves in the inflammatory membrane. Ablation of these nerves will decrease discogenic pain

with over 10-year follow-up. It is not unusual to have patients returning for consultation 20 years later, reporting their satisfaction of their endoscopic decompression and returning with conditions of lumbar spondylosis and stenosis secondary to the aging process.

It is very common for patients to return after years of successful alleviation of their symptomatic condition ranging from 2-20 years following their successful endoscopic surgery. Video examples of endoscopic procedures and playlists of various conditions are demonstrated on you tube at the DISC website www.sciatica.com. The disruptive nature of endoscopic spine surgery, when performed at a 
Citation: Yeung AT (2018) Lessons Learned from 27 Years' Experience and Focus Operating on Symptomatic Conditions of the Spine under Local Anesthesia: The Role and Future of Endoscopic Spine Surgery as a "Disruptive Technique" for Evidenced Based Medicine. J Spine 7: 413. doi: 10.4172/2165-7939.1000413

Page 5 of 5

high level by true experienced experts, is one disruptive innovation that should gain interest as part of the future of safe and cost-effective spine care.

\section{Conclusion}

Operating under local anesthesia makes endoscopic procedures exceeding safe. It also helps the surgeon use his ever-growing experience to better select and stratify his patients for endoscopic treatment to the extent that the experience allows the surgeon to predict his results as soon as the endoscopic procedure is finished.

The future of endoscopic mediated surgery will be a safe, efficient, and cost-effective method and technique that will bridge the huge gap between naturopathic and nonsurgical remedies favored by and paid for out of pocket by many patients. In the very severe degenerative painful conditions where surgeons are reluctant or unable to offer help because age and co-morbidities, endoscopic procedures will also bridge the gap between pain management and surgically morbid procedures designed for deformity and instability. The "surgeon factor" is critical for any individual to be able to attain the skills and experience in order to be adept at both ends of the spectrum. It will take approximately 5 years for the average trained technician to be skilled and confident enough to adopt endoscopic procedures as a dedicated subspecialty and 10 years to really experience and understand the role and Future of endoscopic philosophy and techniques.

\section{References}

1. Chou R, Deyo R, Friedly J, Skelly A, Weimer M, et al. (2017) Systematic pharmacologic therapies for low back pain: A systematic review of an American College of Physicians clinical practice guideline. Ann Intern Med 166: 480-492

2. Al-Tamimi $\mathrm{P}$ (2017) The functional and economic outcome of lumbar discectomy:
A comparative study of fenestration discectomy versus hemilaminectomy and discectomy. J Spine 6: 1-8.

3. Yeung AT (2018) The Yeung Percutaneous Endoscopic Lumbar Decompressive Technique (YESSTM). J Spine 7: 408.

4. Yeung AT, Kotheeranurak V (2018) Transforaminal endoscopic decompression of the lumbar spine for stable degenerative spondylolisthesis as the least invasive surgical treatment using the YESS surgery technique. J Spine 7: 407.

5. Yeung AT (2017) In-vivo endoscopic visualization of pain generators in the lumbar spine. J Spine 6: 385

6. Yeung AT (2017) Transforaminal endoscopic decompression for painful degenerative conditions of the lumbar spine: A review of one surgeon's experience with over 10,000 cases since 1991. J Spine Neurosurg 6: 266.

7. Yeung A, Yeung CA (2017) Endoscopic identification and treating the pain generators in the lumbar spine that escape detection by traditional imaging studies. J Spine 6: 369 .

8. Yeung AT, Yeung CA, Salari N, Field J, Navratil J, et al. (2017) Lessons learned using local anesthesia for minimally invasive endoscopic spine surgery. J Spine 6:377.

9. Yeung AT (2017) Endoscopic decompression for degenerative and isthmic spondylolisthesis. J Neurol Disord 5: 371.

10. Yeung AT (2017) The role of endoscopic surgery in the treatment of painfu conditions of an aging spine: State of the Art. J Neurol Disord 5: 372.

11. Yeung AT (2006) Selective endoscopic discectomy ${ }^{\mathrm{TM}}$ twelve years' experience" in Kambin P (ed) Atlas of Arthroscopic and Endoscopic Spinal Surgery, Text and Atlas, (2nd edn), Humana Press, Inc., Totowa, NJ, USA.

12. Yeung AT, Yeung CA, Zheng $Y$ (2010) Endoscopic decompression, ablation and irrigation: A minimally invasive surgical technique for painful degenerative conditions of the lumbar spine. Cosar, Khoo (eds). J Spine Neurosurg 5: 3.

13. Gore S, Yeung AT (2014) The "inside out" transforaminal technique to treat lumbar spinal pain in an awake and aware patient under local anesthesia: Results and a review of the literature. Int J Spine Surg 8: 28. 